\title{
Fault detection and diagnosis of historical vehicle engines using acoustic emission techniques
}

\author{
Alejandro Roda-Buch ${ }^{1,2}$, Emilie Cornet ${ }^{1}$, Guillaume Rapp ${ }^{1}$, Brice Chalançon ${ }^{3}$, Stefano Mischler ${ }^{2}$, Laura \\ Brambilla ${ }^{1}$ \\ ${ }^{1}$ Haute Ecole Arc Conservation-Restauration, HES-SO University of Applied Sciences and arts Western Switzerland, Espace de l'Europe 11, \\ $\mathrm{CH}-2000$, Neuchâtel, Switzerland \\ 2 Ecole Polytechnique Fédérale de Lausanne, EPFL CH-1015, Lausanne, Switzerland \\ ${ }^{3}$ Association de Gestion du Musée National de l'Automobile, 188 Av. de Colmar, 68100 Mulhouse, France
}

\section{ABSTRACT}

The reactivation of artefact mechanisms is always a challenge for conservators. Non-invasive diagnostic techniques, applicable directly on the artifacts, allows for performing early-stage diagnostics and avoiding damage. The Acoustic Emission Monitoring of Historical Vehicles (ACUME_HV) project represents the first use of acoustic emission (AE) as a non-invasive technique for the diagnostics of historical vehicles. The aim of this project is to develop an objective, human-independent method. This will help museum personnel to make decisions regarding the reactivation of historical vehicle engines using measurements and data analysis rather than merely personal experience. Herein, we present the results of the first phase of the ACUME_HV project, which was focused on the development of a protocol for the use of $\mathrm{AE}$ during cold tests.

\section{Section: RESEARCH PAPER}

Keywords: Historical vehicles; acoustic emission; NDT; fault diagnosis

Citation: Alejandro Roda-Buch, Emilie Cornet, Guillaume Rapp, Brice Chalançon, Stefano Mischler, Laura Brambilla, Fault detection and diagnosis of historical vehicle engines using acoustic emission techniques, Acta IMEKO, vol. 10, no. 1, article 11, March 2021, identifier: IMEKO-ACTA-10 (2021)-01-11

Editor: Eulalia Balestrieri, University of Sannio, Italy

Received May 1, 2020; In final form September 26, 2020; Published March 2021

Copyright: This is an open-access article distributed under the terms of the Creative Commons Attribution 3.0 License, which permits unrestricted use, distribution, and reproduction in any medium, provided the original author and source are credited.

Funding: This work was supported by HES-SO RCDAV funding, Switzerland

Corresponding author: Laura Brambilla, e-mail: laura.brambilla@he-arc.ch

\section{INTRODUCTION}

The peculiarity of the objects constituting technical, industrial, and scientific cultural heritage lies in the presence of specific mechanisms. The functionality of these artefacts, i.e. the possibility of making them work, is an integral part of the objects themselves. The reactivation of these mechanisms, however, is often a challenge for the conservators, especially if the functioning has been stopped for a long period of time. In short, the presence of corrosive products, deposits, oxidation, and particles, as well as the scaling of oils or lubricants, can prevent the mechanisms from working properly or can even lead to their breakdown during reactivation. In addition, the reactivation procedure must be performed while respecting the material authenticity of the object, i.e. preserving the original parts of the mechanism as far as possible.

Among the objects that incorporate mechanisms, vehicles are especially complex due to of the number of parts and or sub- systems involved in their functioning. Given the aforementioned obstacles to the reactivation of historical engines, the choice of exhibition and maintenance of the vehicles in museums and collections is generally reduced to the following two options:

i. $\quad$ static: the vehicle is exposed and its engine is never operated (on occasion, the engine is even removed from the vehicle for practical and safety reasons);

ii. dynamic: the vehicle is used, or at least activated periodically.

In order to be able to reactivate the mechanisms without damage while simultaneously preserving the cultural value and the original materials of the objects, the conservators require specific diagnostic tools for the detection of any malfunctions at a very early stage.

In the field of historical vehicle conservation, there currently exists no reliable non-invasive technique for the diagnosis and monitoring of the engine's functioning. Therefore, the maintenance of old engines is extremely time consuming and 
requires personnel with specialised competency. Indeed, the assessment of the correct functioning is generally left to the expertise of the specialists, who tend to largely base their evaluation on previous experience, using methods ranging from visual examination to listening to the sound of the engine. However, this approach is empirical, human-dependent, and has never been correctly standardised.

As such, the use of non-invasive techniques, such as acoustic emission (AE) techniques [1], for the evaluation of the mechanisms' condition could prevent damage occurring during the reactivation and could help in developing a monitoring protocol based on factual measurements.

The Acoustic Emission Monitoring of Historical Vehicles (ACUME_HV) project, led by HE-Arc CR, represents the first use of $\mathrm{AE}$ as a non-invasive technique for the diagnostics of historical vehicles, and was carried out in collaboration with the Musée National de l'Automobile de Mulhouse (MNAM) in France. The aim of this project is to develop an objective, human-independent method that will assist museum personnel with their decision making regarding the reactivation of historical vehicle engines using measurements and data analysis rather than personal experience alone.

The use of the AE technique has already been applied to the diagnosis of faults during the operation of newly produced engines [2]-[10]. Here, El-Ghambry et al. [4] analysed the AE rms signals of diesel engines in the time domain in view of identifying - using the machine timing - machine fault conditions. The authors used selective time windowing of the AE signals and different statistical features and pattern recognition techniques to isolate and identify various fault conditions in reciprocating engines.

Meanwhile, Douglas et al. [5] studied the interaction between piston rings and cylinder liners using $\mathrm{AE}$ for in-service engine monitoring and found that, for small high-speed direct injection (HSDI) engines, the AE activity was proportional to the piston speed and that the most likely AE source (among other contributions) was the boundary friction between the oil-control ring and the cylinder liner.

Various analytical models and predictive tools [11]-[19] have been developed through using the AE technique to characterise and model the friction and wear in sliding contacts under various lubricated conditions, including the scuffing phenomenon.

Meanwhile, in the field of cultural heritage, the AE technique has been used for monitoring the conservation state of heritage objects or for detecting insects in wooden musical instruments [20]-[22]. In addition, AE has been used for several years for the structural damage analysis and health monitoring of historical masonry buildings. Of particular interest in this domain is the application of the Gutenberg-Richter (GBR) law to AE measurements in order to correlate the magnitude and the number of events within a certain time period [23]-[26].

Despite the extended use of the AE technique in the field of modern engine diagnosis, the capacity of this technique for providing valuable diagnostic results remains a challenge in the field of cultural heritage. In short, the attendant limitations arise from the technical characteristics of the historical vehicles and their conservation state, mainly in the case of reactivation processes.

The present work is organised in terms of five sections. In section 2, the methodology adopted for the ACUME_HV project to define a protocol for the reactivation of the historical vehicle engines is described before section 3 describes the measuring system and the set of tests carried out to follow the discussed methodology. In section 4, the results accounting for the non-uniform driving speed of the engine during the tests are presented and discussed as is a comparison between the tests to discriminate the effect of compression in the cylinders. Finally, the main conclusions are summarised in section 5 .

\section{METHODOLOGY}

A reliable diagnostic tool must have the capacity to capture signal features that can be correlated with the state of the system. The conservation/restoration procedure for reactivating an engine [27] begins with a visual inspection of the whole engine in view of evaluating the general condition of the individual components. At this stage, the liquids of the oil and cooling systems are changed and the accessorial components are repaired where necessary. Prior to starting the engine, the next step involves assessing the functioning of the mechanisms. In order to achieve this, the engine is moved manually before an expert checks the engine's operation, both visually and acoustically. This procedure is known as a 'cold test' and is performed without starting the engine to minimise the possible damage during the reactivation process. To ensure this condition, the combustion process is excluded from the evaluation, with only the mechanical displacements taken into account [27].

At this critical stage, the procedure developed during the ACUME_HV project will be introduced in view of replacing the human-dependent diagnosis, with the measurement of the AE signals generated by friction and/or impacts at different contact pairs of the engine mechanisms, such as crankshaft/connecting rod, connecting rod/piston, piston rings/cylinder liner, as well as inside the cam chain system. The airflow or air leakage at the valve inlets/outlets and between the piston-ring/cylinder-liner gaps can also be detected at this point. The following step of this new procedure involves obtaining the mechanical signature of the engine for different reactivating conditions (i.e. with/without cylinder compression). Finally, the last step involves the use of statistical analysis techniques to extract specific features that will then be used to classify and correlate the operational state of the mechanical parts of the engine.

First, the method was tested on an engine that was removed from the vehicle to ensure complete access to all the parts of the engine. This engine, known as a 'bench engine', was bought by the MNAM as a spare for the possible reparation of certain vehicles in their collection. The engine was mounted on a test bench while keeping all of the cam system and pump mechanisms operative. The mechanical parts were manually operated at a relatively low rotating speed through turning the crankshaft via a handle. This procedure allowed for maintaining good control of the interplay between different parts, thus avoiding possible damage to the machine.

\section{ENGINE, MEASUREMENT SYSTEM AND TEST SETS}

The AE tests were performed on two Renault AG1-type engines (Figure 1), which are part of the collection held by the Musée National de l'Automobile de Mulhouse. Among the reasons for selecting this specific model were the easy access to most parts of the engine, even when assembled in the vehicle, the simplicity of the engine, which has only two cylinders and basic auxiliary systems, and the availability at MNAN of three identical engines (AG1), two of which are still in working order [28].

The measurement equipment is shown in Figure 2a, while a block diagram of the experimental set-up is schematised in 
Figure 2b. An AE system from Vallen ${ }^{\circledR}$ was used to acquire the AE signals, which included a MB2-V1 chassis with four AE sensors and four parametric input channels. The four broadband AE sensors were VS900-M (between 100 and $900 \mathrm{kHz}$ ) sensors, with the corresponding AEP5 preamplifiers $(+34 \mathrm{~dB})$ also included in the system. The sampling frequency of the AE signals was set to $2 \mathrm{MHz}$, while a $1-\mathrm{MHz}$ low pass filter was used to reduce the noise from high frequencies. The crankshaft angular position was also measured with a full continuous $360^{\circ}$ smart position sensor (VISHAY Spectrol 601-1045; output signal 0$5 \mathrm{~V}$ ) at a sampling rate of $1.25 \mathrm{kHz}$.

To carry out this research, three different sets of tests were implemented. An initial set of measurements was performed to determine the optimal location of the AE sensors for obtaining representative signals from different AE sources [29], [30]. The locations selected for the following sets of tests were those presenting higher signal levels, lower signal-to-noise ratios, and the highest presence of events for the measured AE signals. Figure 3 shows the final locations of the four AE sensors. Here, two were placed on the cylinder block, the first (1) on the outer part of the first cylinder (the one closest to the front), and the second (2) on the opposite side close to the first cylinder valves. Meanwhile, the remaining two sensors were placed on the crankcase, one (3) on the cover of the gears of the cam system, and the other (4) on the crankcase leg of the valve side.

A second set of tests was then conducted to analyse the effect of the engine speed on the AE signal level. In this set, the spark plugs of both cylinders were removed to prevent air compression inside the cylinders and, consequently, to produce a smoother and more constant motion of the engine. Here, different engine speeds were tested, ranging from 0.25 to 1.2 cycles per second (cps), with the engine activated manually using an external handle.

Finally, a third set of tests was performed to observe the influence of the compression of the air inside the cylinders on the AE signals. The results obtained with the spark plugs removed (set 2) were then compared with those obtained with the plugs left in (set 3).

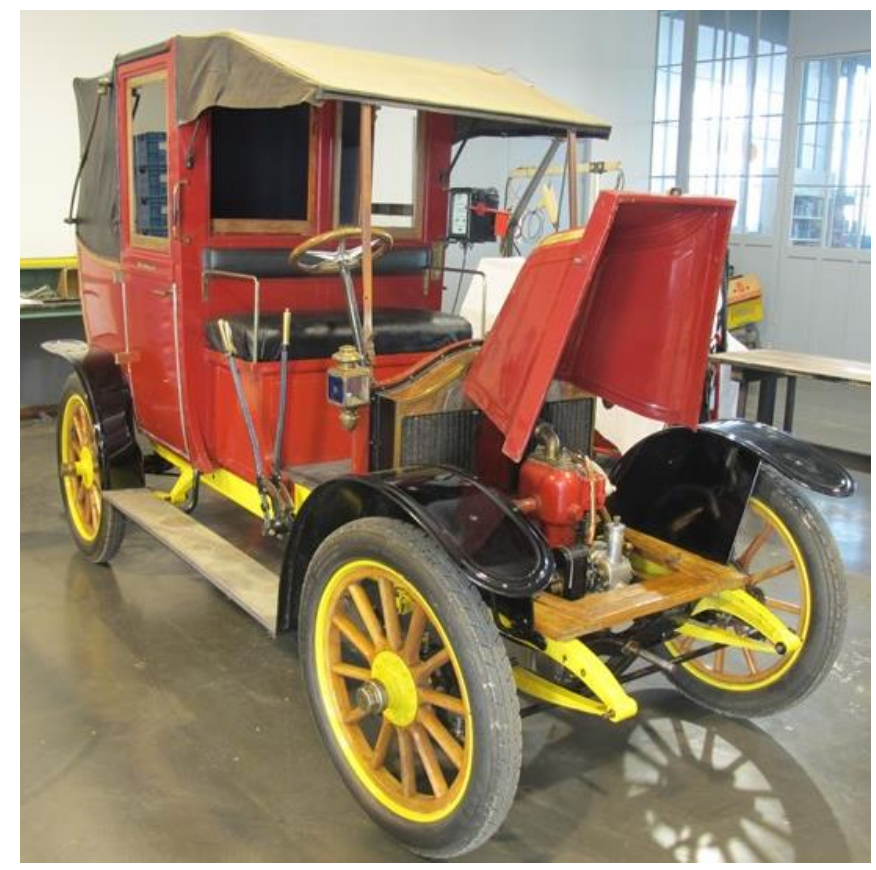

Figure 1. Renault AG1 (Inv.2209) with its 2 cylinder engine (C MNAM, 2016).
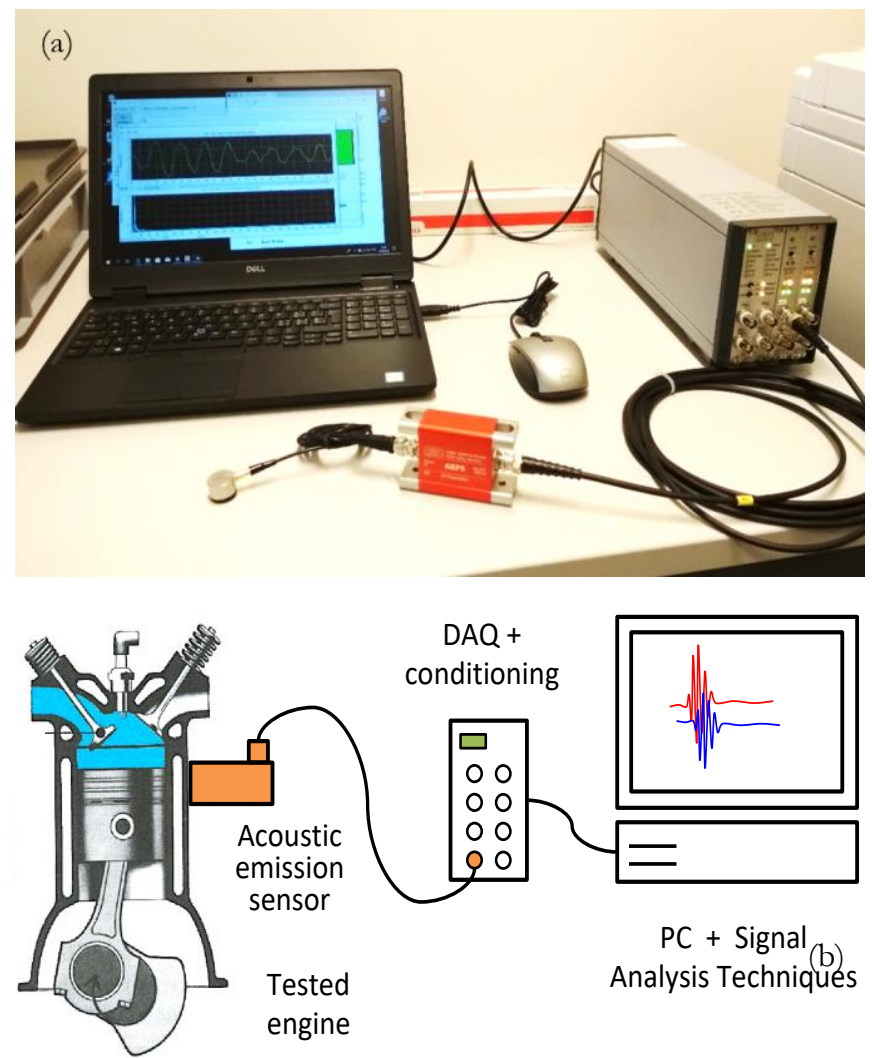

Figure 2. (a) Vallen ${ }^{\circledR}$ measurement system of AE signals, (b) block diagram of the experimental set-up (C) HE-Arc CR, 2018).

Test sets 2 and 3 were also performed on two different engines to observe the main characteristics of the mechanical signature and the mechanical airflow signature of different motors of the same type, that is, the bench engine already used for test set 1 and the engine of the collection car, which remained mounted in the car.

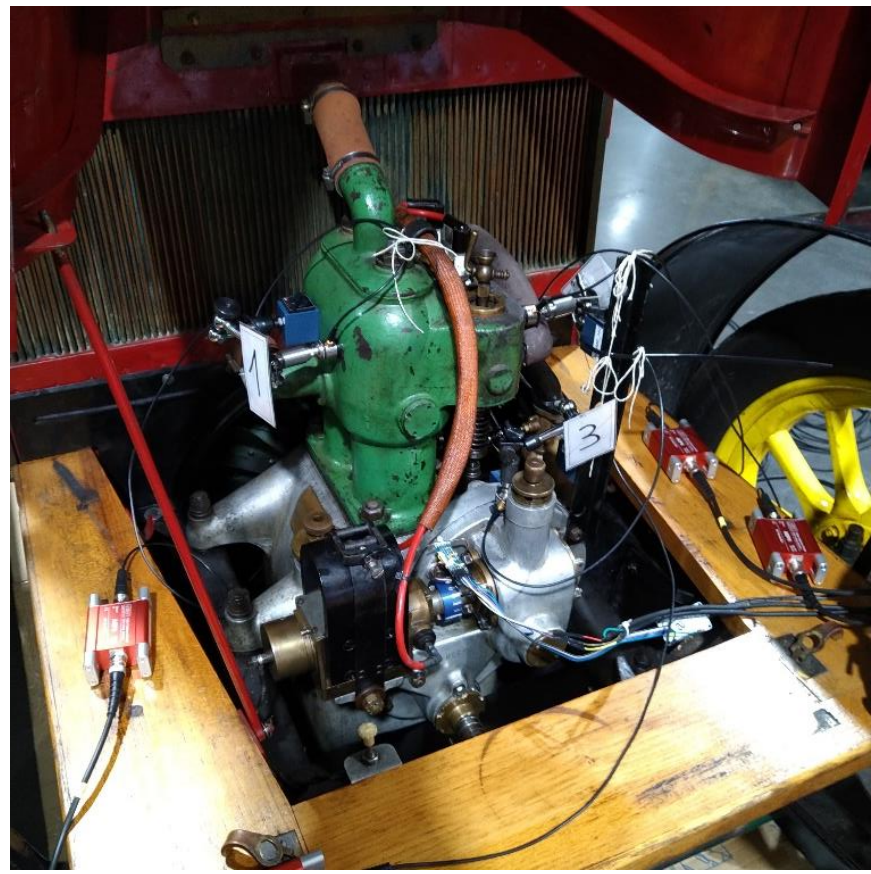

Figure 3. AE sensor location on the Renault AG1 engine (C HE-Arc CR, 2019). 
(a)

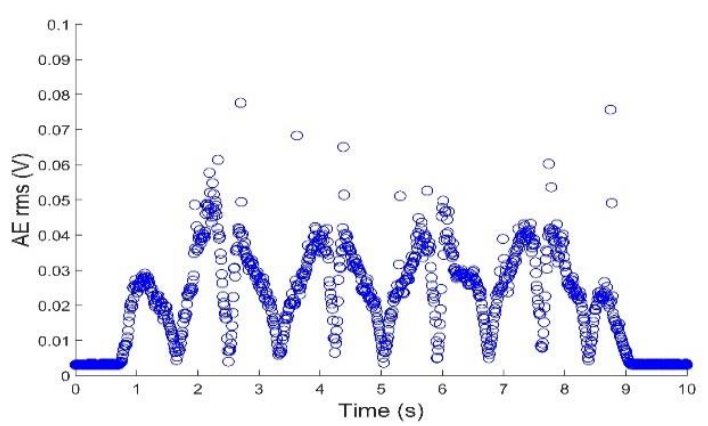

(b)
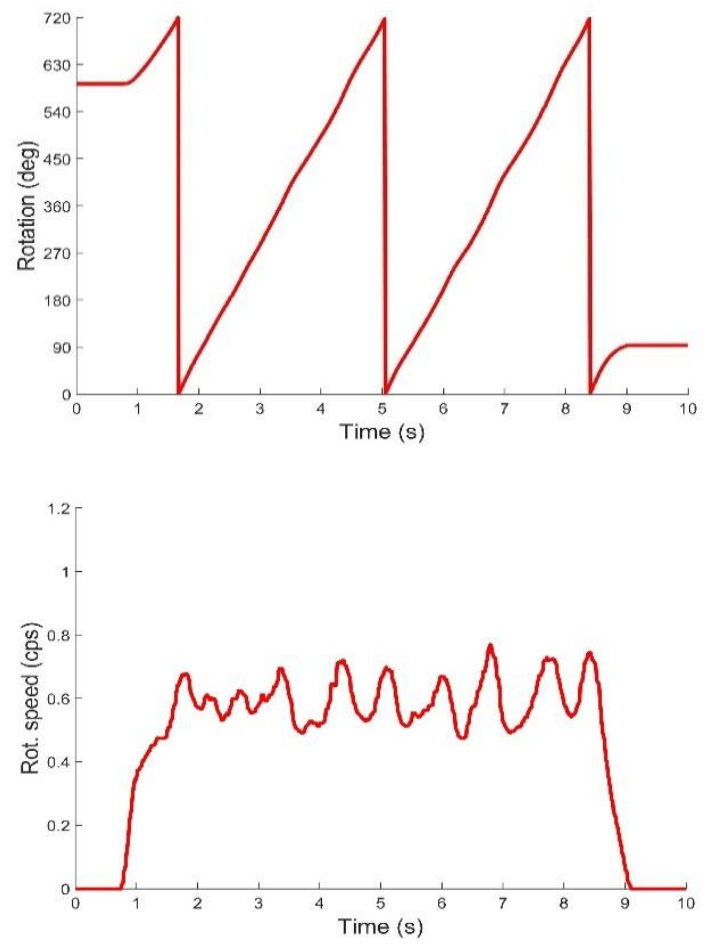

(c)

Figure 4. Time evolution of (a) AE rms signal of sensor 1, (b) crankshaft angular position, (c) crankshaft rotational speed.

\section{RESULTS}

In this section, the results for two specific studies are presented. The first study relates to the influence of the rotational speed of the engine on the $\mathrm{AE}$ feature and the second to the mechanical signature and the mechanical airflow signature of the two engines.

The root means square of the AE signals (AE rms) was the feature used to extract the mechanical and air compression signature of the engine under cold-test conditions. The rms was calculated for blocks of signals with a rate of $10 \mathrm{~ms}$. This feature is related to the AE signal energy generated by, for example, the sliding friction processes in contact pairs [6].

\subsection{Engine speed influence in acoustic emission signal feature}

Figure $4 \mathrm{a}$ shows the time change of the AE rms of the cylinder at sensor 1, Figure $4 \mathrm{~b}$ the angular position, and Figure $4 \mathrm{c}$ the rotational speed of the crankshaft. In Figure $4 \mathrm{~b}$, the crankshaft angular position is related to the thermodynamic cycle of the engine, which is comprised of $720^{\circ}$ or two complete crankshaft revolutions. When the first piston was at the top dead centre (TDC) position, the rotation reached angular values of 0 $\circ, 360^{\circ}$ or $720^{\circ}$. It can be seen that the test started slightly before the piston passed by the TDC before the crankshaft was rotated by four revolutions and its movement was extended for a little longer. This procedure guaranteed that two thermodynamic cycles were fully recorded. Figure $4 \mathrm{c}$ was obtained using the time derivative of the curve in Figure 4b.

It was clear that there was a high correlation between the AE rms feature and the crankshaft angular position and, consequently, the relative motion of the piston to the cylinder. Minimum AE rms values emerged at the TDC and the bottom dead centre (BDC) of both pistons where the piston speed was the lowest, while maximum values emerged at the mid position between the TDC and BDC where the piston speed was maximised. This indicates that the main source of the AE waves was the sliding friction between the piston rings and the cylinder liners. Another outcome that must be highlighted is that the crankshaft rotational speed was not constant throughout the test (Figure 4c), which was due to the way the motion was driven (i.e. manually) and to the variable resistance torque of the engine during its motion.

A normalisation of the $\mathrm{AE}$ rms feature to a defined crankshaft rotational speed of $0.5 \mathrm{cps}$ was performed in order to compare the AE rms feature for different crankshaft positions obtained at different speeds with both the same test and with different tests. This speed was selected since it can be easily reached in cold tests both with and without air compression inside the cylinders. To transform the AE rms from the measured signal to the normalised speed, a proportional relationship described by Eq. 1 was applied at regular time intervals of $10 \mathrm{~ms}$ :

$$
A E_{\text {rms,norm }}(t)=A E_{r m s, \text { meas }}(t) \frac{0.5 c p s}{n_{\text {meas }}(t)}
$$

where $A E_{\text {rms,norm }}(t)$ and $A E_{\text {rms,meas }}(t)$ are the $\mathrm{AE}$ features normalised and measured, respectively, and $n_{\text {meas }}(t)$ and 0.5 are the measured crankshaft rotational speed (in cps) and the normalised speed for the corresponding time interval, respectively. This transformation is based on the results obtained by Douglas et al. [5], which indicated that the AE energy generated during the sliding friction between the piston rings and the cylinder liners is proportional to the relative speed between the solids in contact. This result was confirmed, for the range of speeds tested, by observing the AE rms from measured signals as a function of the speed at different crankshaft positions (see Figure 5).

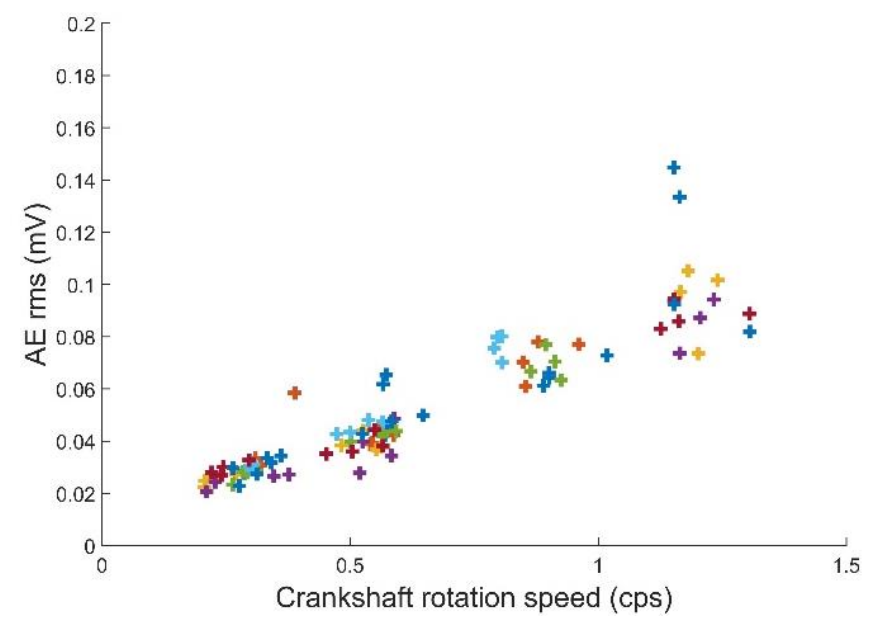

Figure 5. AE rms signal of sensor 3 vs. crankshaft rotational speed at $\pm 75^{\circ}$ from TDC. 


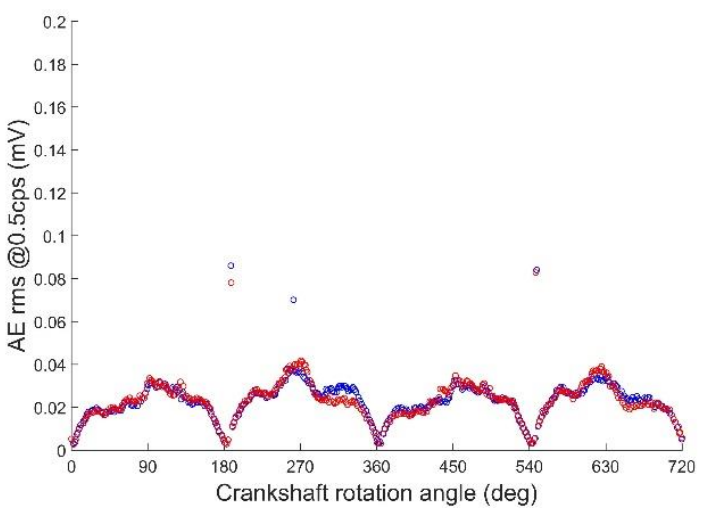

(a)

Figure 6. Normalised AE rms signals of sensor 1 with spark plugs removed: (a) bench engine, (b) collection engine.

\subsection{Acoustic emission signatures}

The normalised AE rms signals measured using cylinder sensor 1 in relation to the crankshaft angular position are plotted in Figure 6 for the two different engines: (a) the bench engine and (b) the collection car engine.

The crankshaft rotation evolved for two complete thermodynamic cycles $\left(0^{\circ}-720^{\circ}\right.$; first cycle in blue and second cycle in red) corresponding to four full revolutions of the crankshaft. The TDC of cylinders 1 and 2 corresponded to a $0^{\circ}$ and a $180^{\circ}$ rotation, respectively. Both pistons completed four back-and-forth reciprocating motions, while every pass through the TDC and the BDC corresponded to a change in speed direction, where the piston speed was null. At these points, the $\mathrm{AE}$ rms was close to zero, which confirmed that the main source of the $\mathrm{AE}$ events in the cylinder block was the sliding friction between the piston rings and the cylinder liner. The absence of air compression inside the cylinders prevented, in these cases, the generation of $\mathrm{AE}$ waves from airflows.

The results are clearly reproducible for every engine when the normalised AE rms is taken into account. These results represent the mechanical signature (with an absence of air compression) of the engine's condition. In fact, when comparing the AE rms evolution between the two engines, it became clear that the mechanical signature is univocally representative of the engine.

The normalised AE rms signals of sensor 1 when the spark plugs were left in, meaning air was compressed inside the

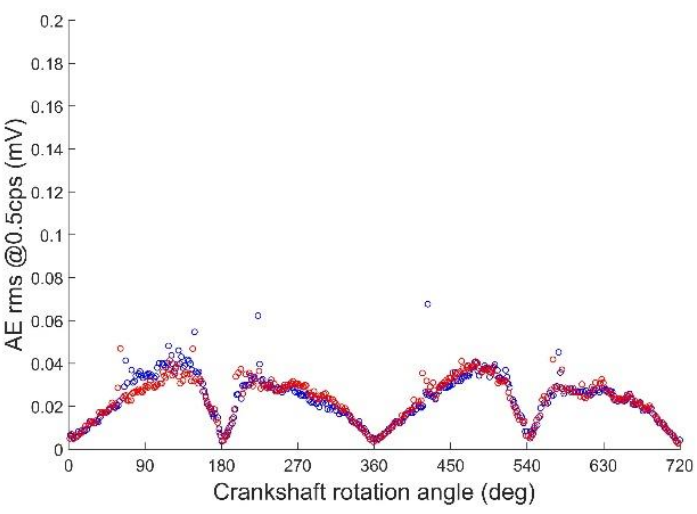

(b)

cylinders, are plotted in Figure 7 for the two previously tested engines.

Two main differences can be observed for the results with (Figure 7) and without (Figure 6) spark plugs. First, there was a clear increase in $\mathrm{AE}$ rms level in the range of $90^{\circ}-180^{\circ}$ and 630 ${ }^{\circ}-720^{\circ}$, which was associated with the compression phase of the second and first cylinder, respectively. Second, various punctual spiky AE events that were related to the valves' opening and closing operations emerged. The AE rms signals shown in Figure 7 are the combination of the mechanical signature (Figure 6) and the $\mathrm{AE}$ events from the airflows in the compression phases and the valve operation of the engine when the spark plugs were left in.

A deeper analysis of the data shown in Figure 7 allowed for the detection of a failure in the valve system of the collection engine (case b). The AE rms signals of the bench engine (Figure $7 \mathrm{a})$ during the compression phases of both cylinders $\left(90^{\circ}-180^{\circ}\right.$ and $630^{\circ}-720^{\circ}$ for the second and first cylinders, respectively) presented a similar evolution. On the contrary, for the collection engine (Figure $7 \mathrm{~b}$ ), the signal evolution of the compression phase of the second cylinder $\left(90^{\circ}-180^{\circ}\right)$ was significantly different (in both shape and level) from the signal of the compression phase of the first cylinder $\left(630^{\circ}-720^{\circ}\right)$. After noting this divergent compression evolution, a specific test was performed to check the airtightness of the second cylinder, which revealed a fault in the seat of the intake valve, leading to a loss in compression of around $35 \%$.

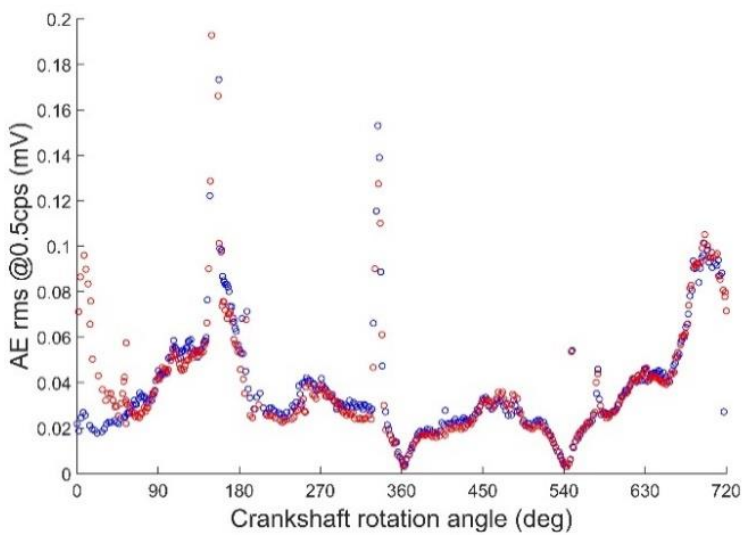

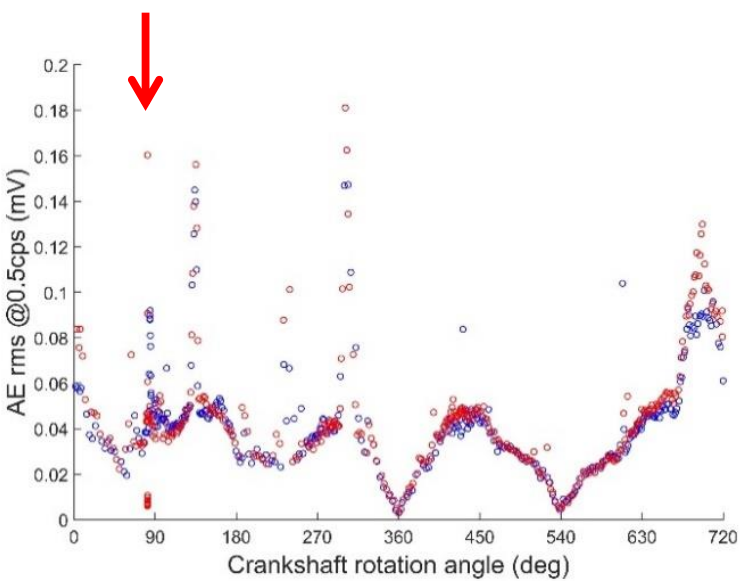

(b)

Figure 7. Normalised AE rms signals of sensor 1 with spark plugs left in: (a) bench engine, (b) collection engine. 

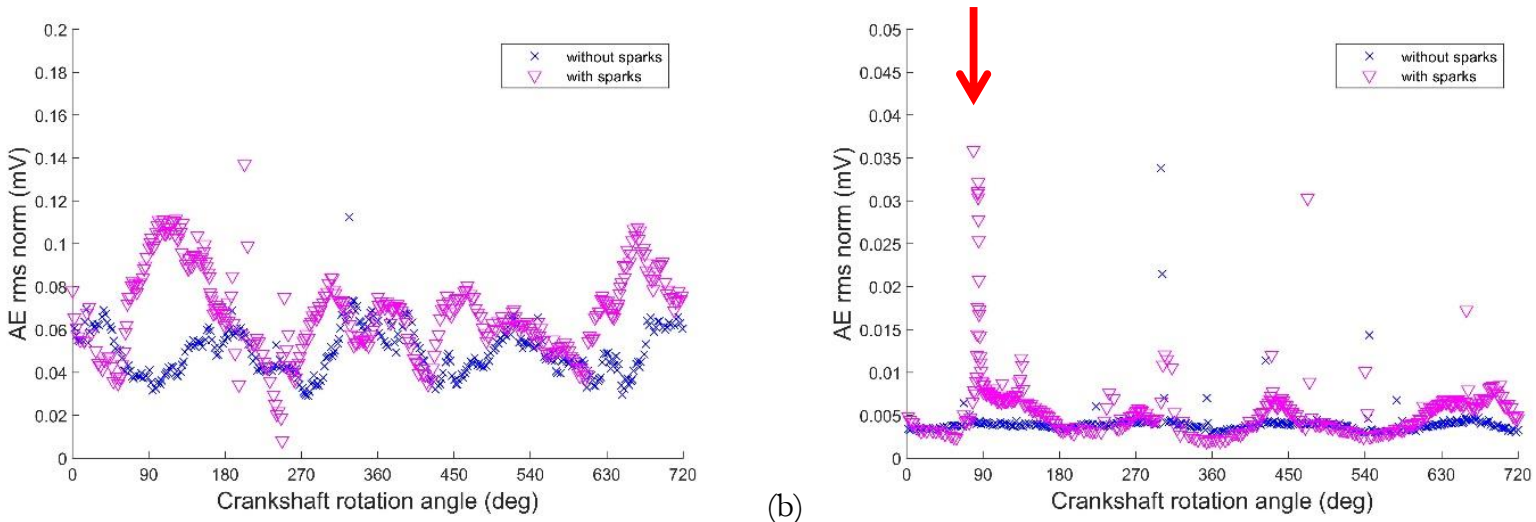

Figure 8. Normalised AE rms signals of sensor 3: (a) bench engine, (b) collection engine.

This malfunctioning behaviour could also be observed when analysing the AE rms signals of other channels. Figure 8 shows the normalised AE rms evolution for the bench (case a) and collection (case b) engines. Here, the results without (in blue) and with (in red) spark plugs are superimposed for each engine. On comparing the red results in both graphs, it was clear that while the signal evolution during the compression phase for the bench engine was quite similar, the signal of the collection engine presented a spike at around $90^{\circ}$ (marked with arrows in Figure $7 \mathrm{~b})$ during the compression phase of the second cylinder. This event was not observed either in the compression phase of the first cylinder of the collection engine or in the compression phases of the bench engine.

\section{CONCLUSIONS}

In this paper, a diagnostic tool based on $\mathrm{AE}$ measurements was developed to help conservation/restoration specialists in reactivating historical vehicle engines. This new tool will provide innovative and more objective diagnostic features that are free of human subjective evaluation. By performing cold tests at the initial reactivation stages to prevent any damage to the heritage artefacts, the mechanical signature of the engine's condition can be obtained from appropriate AE measurements.

In order to collect reliable AE signals for various types of engine, a preliminary study should be performed in view of determining the correct sensor positions, which will ensure that appropriate signatures for performing an accurate diagnosis of the engine's condition will be obtained.

When using AE rms features, the influence of the crankshaft rotational speed in the signal levels must be taken into account. In this work, for the range of speeds used in the tests, a proportional correlation was applied to normalise the AE rms signal. This normalisation procedure ensured good reproducibility of the obtained signals. The use of a controlled speed system for driving the engine motion or the use of other analytical techniques in the frequency or time-frequency domains could help to avoid the issue of non-constant rotational speeds.

The preliminary results demonstrated that performing cold tests using this $\mathrm{AE}$ technique is a promising approach to detecting various malfunctions (e.g. air leakages in the valves), one that involves applying adequate data treatment procedures to the AE features obtained from the mechanical signatures of the engines.

Another interesting potential use for this technique is in the future maintenance procedures of the analysed engines. Once a reliable signature of the actual working condition of an engine is registered, this diagnostic tool can be used to compare or analyse the evolution of the future engine signatures in relation to the reference signature. As such, a database of the engines of an existing collection could be created and stored for future comparisons.

\section{ACKNOWLEDGEMENTS}

The authors wish to acknowledge HES-SO RCDAV for funding the ACUME_HV project, the Musée Nationale de l'Automobile in Mulhouse (France) for their material and technical support, and Jean-Charles Peruchetti, lecturer at ENSISA in Mulhouse (France), for the development of the engine's angular speed monitoring sensor.

\section{REFERENCES}

[1] C. B. Scruby, An introduction to acoustic emission, J. Phys. E: Sci. Instrum. 20 (1987), pp. 946-953.

DOI: $10.1088 / 0022-3735 / 20 / 8 / 001$

[2] S. Delvecchio, P. Bonfiglio, F. Pompoli, Vibro-acoustic condition monitoring of internal combustion engines: a critical review of existing techniques, Mech. Syst. and Signal Proc. 99 (2018), pp. 661-683. DOI: 10.1016/i.vmssp.2017.06.033

[3] B.C. Kaul, B. Lawler, A. Zahdeh, Engine diagnostics using acoustic emissions sensors, SAE Int. J. Engines 9(2) (2016), pp. 684-692.

DOI: $\underline{10.4271 / 2016-01-0639}$

[4] M. H. El-Ghambry, R. L. Reuben, J. A. Steel, The development of automated pattern recognition and statistical feature isolation techniques for the diagnosis of reciprocating machinery faults using acoustic emission, Mech. Syst. and Signal Proc. 17(4) (2003), pp. 805-823.

DOI: $10.1006 / \mathrm{mssp} .2002 .1473$

[5] R. M. Douglas, J. A. Steel, R. L. Reuben, A study of the tribological behaviour of piston ring/cylinder liner interaction in diesel engines using acoustic emission, Tribology International 39 (2006), pp. 1634-1642.

DOI: 10.1016/i.triboint.2006.01.005

[6] P. Nivesrangsan, J. A. Steel, R. L. Reuben, Source location of acoustic emission in diesel engines, Mechanical Systems and Signal Processing 21 (2007), pp. 1103-1114.

DOI: $\underline{10.1016 / \text { i.ymssp.2005.12.010 }}$

[7] S. Johansson, P. H. Nilsson, R. Ohlsson, B.-G. Rosén, Experimental friction evaluation of cylinder liner/piston ring 
contact, Wear 271 (2011), pp. 625-633.

DOI: $10.1016 /$ j.wear.2010.08.028

[8] J.-D. Wu, C.-H. Liu, An expert system for fault diagnosis in internal combustion engines using wavelet packet transform and neural network, Expert Syst. Appl. 36 (2009), pp. 4278-4286. DOI: $10.1016 /$ j.eswa.2008.03.008

[9] N. Wei, J.X. Gu, F. Gu, Z. Chen, G. Li, T. Wang, A.D. Ball, An investigation into the acoustic emissions of internal combustion engines with modelling and wavelet package analysis for monitoring lubrication conditions, Energies 12 (2019), pp 640 659.

DOI: $10.3390 /$ en 12040640

[10] M. Shuster, D. Combs, K. Karrip, D. Burke, Piston Ring Cylinder Liner Scuffing Phenomenon Studies Using Acoustic Emission Technique, SAE International, Warrendale, PA, USA, 2000 DOI: $\underline{10.4271 / 2000-01-1782}$

[11] Y. Fan, F. Gu, A. Ball, Modelling acoustic emissions generated by sliding friction, Wear 268 (2010), pp. 811-815. DOI: $10.1016 /$ j.wear.2009.12.010

[12] Z. Genga, D. Puhanb, T. Reddyhoff, Using acoustic emission to characterize friction and wear in dry sliding steel contacts, Tribology International 134 (2019), pp. 394-407. DOI: $10.1016 /$ i.triboint.2019.02.014

[13] A. Hase, H. Mishina, M. Wada, Correlation between features of acoustic emission signals and mechanical wear mechanisms, Wear 292-293 (2012), pp. 144-150.

Doi: $10.1016 /$ i.wear.2012.05.019

[14] H.S. Benabdallah, D.A. Aguilar, Acoustic emission and its relationship with friction and wear for sliding contact, Tribology Transactions 51(6) (2008), pp. 738-747. DOI: $10.1080 / 10402000802044324$

[15] V. E. Rubtsov, E. A. Kolubaev, A. V. Kolubaev, V. L. Popov, Using acoustic emission for the analysis of wear processes during sliding friction, Technical Physics Letters 39(2) (2013), pp. 223225.

DOI 10.1134/S1063785013020235

[16] D. Baccar, D. Söffker, Wear detection by means of wavelet-based acoustic emission analysis, Mechanical Systems and Signal Processing 60-61 (2015), pp. 198-207. DOI: $10.1016 /$ i.ymssp.2015.02.012

[17] D. Bianchi, E. Mayrhofer, M. Gröschl, G. Betz, A. Vernes, Wavelet packet transform for detection of single events in acoustic emission signals, Mech. Syst. Signal Process. 64-65 (2015), pp. 441-451. DOI: $10.1016 /$ j.ymssp.2015.04.014

[18] F. Saeidi, S. A. Shevchik, and K. Wasmer, Automatic detection of scuffing using acoustic emission, Tribology International 94 (2016), pp. 112-117. DOI: $10.1016 /$ i.triboint.2015.08.021

[19] S. A. Shevchik, F. Saeidi, B. Meylan, K. Wasmer, Prediction of failure in lubricated surfaces using acoustic time-frequency features and random forest algorithm, IEEE Transactions on
Industrial Informatics 13(4) (2017), pp. 1541-1533.

DOI: $10.1109 /$ TII.2016.2635082

[20] D. Thickett, C. S. Cheung, H. Liang, J. Twydle, R. G. Maev, D. Gavrilov, Using non-invasive non-destructive techniques to monitor cultural heritage objects, Insight 59(5) (2017), pp. 230 234.

DOI: 10.1784 /insi.2017.59.5.230

[21] S. Le Conte, S. Vaiedelich, J.H. Thomas, V. Muliava, D. de Reyer, E. Maurin, Acoustic emission to detect xylophagous insects in wooden musical instruments, Journal of Cultural Heritage 16(3) (2015), pp. 338-343. DOI: $10.1016 /$ j.culher.2014.07.001

[22] A. Cariointeri, S. Invernizzi, G. Lacidogna, F. Accornero, Acoustic Emission monitoring of frescos degradation in a XVIIth Century chapter of the Sacred Mountain of Varallo (Italy), Structural Analysis of Historical Constructions 1-3 (2012), pp. 2340-2348.

[23] D. L. Carnì, C. Scuro, F. Lamonaca, R. S. Olivito, D. Grimaldi, Damage analysis of concrete structures by means of acoustic emissions technique, Composites Part B: Engineering 115 (2017), pp. 79-86. DOI: $10.1016 /$ i.compositesb.2016.10.031

[24] A. Carpinteri, G. Lacidogna, N. Pugno, Structural damage diagnosis and life-time assessment by acoustic emission monitoring, Engineering Fracture Mechanics 74 (2007), pp. 273 289.

DOI: $\underline{10.1016 / \text { j.engfracmech.2006.01.036 }}$

[25] A. Barontini, M. G. Masciotta, L. F. Ramos, P. Amado-Mendes, P. B. Lourenço, An overview on nature-inspired optimization algorithms for structural health monitoring of historical buildings, Procedia Engineering 199 (2017), pp. 3320-3325.

DOI: $10.1016 /$ i.proeng.2017.09.439

[26] C. Scuro, P. F. Sciammarella, F. Lamonaca, R. S. Olivito, D. L. Carni, IoT for structural health monitoring, IEEE Instrumentation \& Measurement Magazine, 21(6) (2018), pp. 4 14. DOI: 10.1109/MIM.2018.8573586

[27] B. Chalançon, Les mesures d'émission acoustique appliquées aux moteurs d'automobiles de collection patrimoniale comme outil de diagnostic avant la remise en fonctionnement, MA Thesis HESSO, Neuchâtel, 2019 (in French).

[28] Keller R. and Garnier P., National Car Museum Schlumpf Collection Mulhouse France, Du Donon (editor). Strasboug, France, 2014, p. 80. ISBN 978-2914856744.

[29] A. Roda-Buch, E. Cornat, G. Rapp, B. Chalançon, S. Mischler, L. Brambilla, Development of a diagnostic tool based on acoustic emission techniques for historical vehicle's engines, Proc. of 31èmes JIFT, 24 - 26 April 2019, Tours, France (In press.)

[30] L. Brambilla A. Roda-Buch, E. Cornet, G. Rapp, B. Chalançon, Diagnostics and monitoring of historical vehicle engines by acoustic emission testing, Proc. of the $9^{\text {th }}$ Interim Meeting of the ICOM-CC Metals Working Group, Metal 2019, Neuchâtel, Switzerland. 